\title{
A Review on Image Inpainting to Restore Image
}

\author{
M.S.Ishi ${ }^{1}$, Prof. Lokesh Singh ${ }^{2}$, Prof. Manish Agrawal ${ }^{3}$ \\ ${ }^{1,2}$ (CSE, TIT, Bhopal) \\ ${ }^{3}$ (CSE, TIT Excellence, Bhopal)
}

\begin{abstract}
With the advent of lots of multimedia instruments in today's world peoples are clicking lots of Picture of theirs and also trying to preserve their past pictures. As the time goes on those pictures got damaged. Image inpainting is technique which tries to recover such images which are damaged. Inpainting is an art of modifying the digital image in such a way that the modifications/alterations are undetectable to an observer who has no idea about the original image. Image Inpainting is used to filling the region which are damaged and want to recover from unwanted object by collecting the information from the more promising neighbouring pixels which will add details to the image such that image have close resemblance to the original image and we are not able to differentiate between original image and the inpainted image. In this paper we are trying to study various technique of image inpainting, where the authors used the multiresoultion technique, wavelet transform, counterlet transform, fragment based image completition etc. to complete the image. We also discuss about the advantages and disadvantages of that technique with respect to efficiency and time limit.
\end{abstract}

Keywords: Image Inpainting, multiresoultion, neighboring pixels, undetectable, wavelet transform.

\section{Introduction}

Image Inpainting or Image completion is technique which is used to recover the damaged image and to fill the regions which are missing in original image in visually plausible way. Inpainting, the technique of modifying an image in an undetectable form, it is art which is used from the ancient year. Applications of this technique include rebuilding of damaged photographs \& films, removal of superimposed text, removal/replacement of unwanted objects, red eye correction, image coding. In image inpainting technique the user first selects the region which we want to recover and then he selects the portion from the source region which is more promising in the sense of matching the information and closely identical to the original image, the selected region is also called as patch. The selected patch is applied to damaged image, after that we get the result which we want. In past the inpainting was performed by two classes of algorithms (i) "diffusion based inpainting" and (ii) "texture synthesis". If we are trying to define the inpainting technique then the first thing come in to mind is that this algorithm try to fill the regions by collecting the information from the available environment of that source region, it is trying to form the image which is nearly identical to the original image and found the close identity to the original image. The image design with this technique is alternative to the original image but this technique is such accurate that the person who is unaware of original image will not able to detect that we have reconstruct the image. This method is called inpainting.

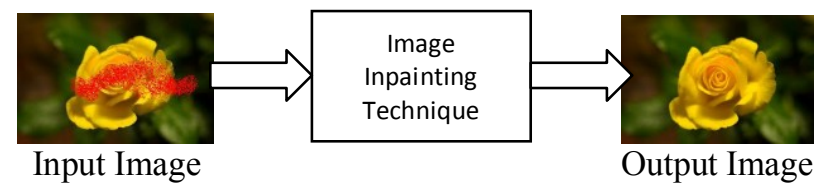

Fig 1 Image Inpainting Method

\section{Basic concept of Image Inpainting}

Inpainting is not only to recover the images which got damaged but also the technique to remove unwanted objects from the image. This technique remove the cracks from the image, fills the missing part from the image, remove the text, dates etc. Inpainting is conceivable by the person who has more idea about this technique or he is specialized in that area or known as artist. Due to manual process it's consume lots of time to give the required result. Image Inpainting could also be called as method which creates alternate image and exploitation of an image. Image inpainting technique creates the original image which is only possible with the help of the information available from the source image/image to recover. In case of digital images, only the available image is taken for the experiment and thus filling in a hole that encompasses a whole object. It is not technique which creates the new image but it try to recover the image which content more promising pixels and that pixels available from the source image and with the help of that we create the clone of the image which is recovered from damaged and the clone of that image is such accurate that if any casual observer going to view 
that image he will detect that image as original image. By using image inpainting along with rebuilding we have to keep this thing in to mind also that image is to recover with maximum accuracy. Image Inpainting not only try to find the texture to be inpainted but also propagate that structure by matching dimensions. Only after matching the dimensions it inserts the patch into the damaged image and then completes the image.

In this paper, different types of image inpainting techniques are discussed. Section 2 discussed about the review on image inpainting techniques. Problems of previous techniques are discussed in section 3. Section 4 is about the conclusion.

\title{
1. Image Inpainting Using PDE
}

\section{Literature Review}

A novel algorithm for digital inpainting of images was done with the help of Partial Differential Equation [1]. That attempts to replicate the basic techniques used by professional rebuilders. In this algorithm the user has to select the area to inpainted and after that algorithm rebuild the image by collecting the information available from the surrounding area of the source image. While filling this region this algorithm considers the way in which isophote lines arriving at the regions' boundaries are completed inside. During completition of image like other algorithm we have no need to provide the surrounding information. This is automatically done, thereby allowing to simultaneously fill-in numerous regions containing completely different structures and surrounding backgrounds. By using the isophote direction this algorithm completed the image.

Advantages: Due to Isophote driven Approach we find the line of equal gray scale values which contains the more promising information and this used to complete the image with less time.

Disadvantage: The main problem with this algorithm is reproduction of large texture regions. This algorithm also unable to recover Partially Degraded Image

\section{Multiresoultion Image Inpainting}

In the multiresoultion approach the damaged image block is divided into equal number of blocks of equal size. After dividing the image, the three threshold values were consider first for the threshold of variance of pixel colors, second and third- for the threshold of percentage [2]. Variance of color pixels has strong indication of containing the details of the image. By using this value we can able to rebuild the image. While rebuilding the image the percentage of damaged pixel was consider. In case the damaged pixel percentage is high, then to inPaint the image we have to consider the global average color. If the percentage is low, in that case we have to consider the information available from the image. After completition of image it evaluates the image with the help of PSNR value. This multilevel PSNR value decides how good the image is inpainted.

\author{
Algorithm \\ 1 a. Let DIB be a damaged image block \\ b. Let a be a threshold of variance \\ c. Let $\beta 1, \beta 2$ be a threshold of percentage, $\beta 1<\beta 2$
}

Algorithm inPaint (block DIB)

2 a. If DIB is a small block then return

Divide DIB into $n^{*} n$ image blocks

b. for each image block IB

Let var be the color variance of IB

Let Mcolor be the mean color of IB

c. if var $<$ a then

\{

let PB be an $x^{*} y$ pixel block in IB

let Ncolor be the mean color of PB

for each $\mathrm{PB}$ in the image block

\{

if the percentage of damaged pixels in $\mathrm{PB}>\beta 2$

inpaint the damaged pixels using Mcolor

else if the percentage of damaged pixels in $\mathrm{PB}>\beta 1$

inpaint the damaged pixels using Ncolor

else

inpaint the damaged pixels using neighbor pixels

\}

d. for each pixel in the boundary of each PB 
3. else

call inPaint(IB)

Advantages: The Single Resolution approach produces the blurred result that overcome by this approach. It also covers the different level of details.

Disadvantages: The issue with this technique is that there is no friendly environment is provided to mark the region which we have to rebuild.

\section{Fragment-Based Image Completition}

In this technique to complete the image we have to remove the background and foreground elements from the image. The parts of the image which are seen with the naked eye can be used as spare parts to repair the image. In this method the first step is to select the region which we want to inPaint for that iterative process is used which selects the region approximately. After that we have to use composite image fragments to complete the image. To select the patch, values of inverse matte is used which gives the high confidence pixels to complete the image and a level is set that provides the incremental approach to travel into the unknown region of image from high to low confidence. In each step we have to select the image fragment from frequently appearing examples. it processed to complete the image with this technique, the image fragments which is composited their probability rise with mean confidence of image.

Process:

Input: image $C$, inverse matte $\mathrm{a}^{-}\left(9\right.$ pixel with $\left.\mathrm{a}^{-}<1\right)$

Output: completed image, $\mathrm{a}^{-}=1$

Algorithm:

1. for each scale from coarse to fine

Approximate image from color and coarser scale

2. Compute confidence map from $\mathrm{a}^{-}$and coarser scale

3. Compute level set from confidence map

4. while $\mu$ (b) $<1-\mathrm{e}$

a. for next position $p$ in level set

Compute adaptive neighborhood $N(p)$

b. search for most similar and frequent match $N(q)$

c. composite $N(p)$ and $N(q)$ at $p$, updating color and $\mathrm{a}^{-}$

5. Compute approximation, confidence map and update level set

Advantage: The Image completed with this approach composition of similar fragment is used which iteratively fills the missing regions. We can apply any method like scaling, transformation to composite the fragment.

Disadvantage: The limitation of this technique is that it has direct proportion with examples available from the global image. If we reach in availability of fragments then we can able to complete the image. The building blocks are required to complete the image from unknown regions to the known regions.

\section{Completition Of Images With Natural Scenes}

This method used to done the completion of images of natural scenery, where the removal of a foreground object creates a hole in the image. In this technique to select a patch from the image we limit the search into the horizontal direction only for that purpose we have to use Fourier transform which gives the distinct vertical line at center, by using this approach we gets the more promising pixels in horizontal direction only [4]. This whole process reduces the effort of searching the portion of image which we require to complete the image. We are able to locate the patch that imposed into the rest of image horizontally. During this method if we are trying to recover the area of slopes then privileges also provided for that image. The grid algorithm is used to complete the image in which the image first completed from the left region and then completed from right side. We fill-up the unknown regions of the matte with grid blocks from the source image.

Advantage: These method Saves lots of time with the help of Fourier transform by limiting the search in horizontal direction only. If we compare the result with other technique then we can get the good result in quick time.

Disadvantage: This Method does not apply the computation over all levels and also search is applied to small regions only not at different levels. 


\section{Graph cut Patch Algorithm}

Zhang et. al [5] created a method to inpaint the image which divided into the three steps, First step is spatial range model is decided to get the direction of selecting the patch from the image.in second step source patch is selected by matching the dimensions of the source patch with target patch and if the dimensions doesn't match in that case we have to adjust the dimensions and then we have to enforce the searching areas into the neighborhood around the previous source patch. Third, to guarantee about the non-blurred result graph cut patch updating algorithm is designed. The quality of result corresponds to the human image recognition after image is completed.

Advantage: As algorithm divided into three steps the blurring of images is greatly reduced in this image. The graph cut patch algorithm performs nonblurring updating in this algorithm.

Disadvantage: In this algorithm we need to take care of filling order as well as patch matching and finally the patch updating.

\section{Criminisis Algorithm}

To recover the old techniques of texture synthesis algorithm and inpainting algorithm used to fill the image gaps, the technique is designed to combined advantages of the two approaches. Exemplar based technique contains the method for reinstalling the both texture and structure [6]. This algorithm divided into the few steps. First step is consisting of finding the source region, target region and finally the patch. In first step we have to find the patch, after finding the patch we have to decide the priorities of that patch because it may be the case that we can found the more patch for the same region with maximum accuracy. In this case we have to calculate the product of the confidence term and the data term. The result of this product will give us the more promising patch and that patch can found the close resemblance with the original image. By finding the patch with maximum priority we have to propagate the structure and texture information. The patch we found it can also called as exemplar means the copy of image. The patch we found has a maximum confidence pixel which minimizes the difference between the original image and the image which is result of the exemplar based technique.

Steps

Extract the manually selected initial front.

Repeat until done:

1 a. Identify the fill front.

b. Compute priorities.

2 a. Find the patch with the maximum priority.

b. Find the exemplar.

c. Copy image data from exemplar to image.

3. Update confidence value.

Advantage: This approach is not only helpful to remove the objects from small scale images but this can be applied with the large scale image also. This approach by combining the two techniques provides us the better results.

Disadvantage: This algorithm does not handle the depth of ambiguities. If this algorithm does not found similar patches for synthesis, we can't get desirable result.

\section{Image Inpainting Using Wavelet Transform}

Dongwook Cho et. al [7] presented the technique with the help of the wavelet transform. Here we expect the best global structure estimation of damaged regions in addition to shape and texture properties. If we consider the fact of multiresoultion analysis, data separation, compaction along with the statistical properties then we have to consider the wavelet transform due to its good image representation quality. Wavelet transform try to satisfy the human visual system (HVS). In this algorithm decomposition of incomplete image is done with the help of wavelet and after that wavelet and scaling coefficients is found. The image inpainting process is applied in the wavelet domain by considering both scaling and wavelet coefficient from coarse to fine scales in the target region.

Advantage: This utilizes inter and intra scale dependency to maintain image structure and texture quality using Wavelet Transform.

Disadvantage: In this algorithm mask for regions are defined manually.

\section{Image Inpainting Using MCA}

Holes present in an image are filled with texture by a new image inpainting technique. Same process applied in cartoon image layer. This algorithm is a direct extension of a recently developed sparse- 
representation-based image decomposition method called MCA (morphological component analysis), designed for the separation of linearly combined texture and cartoon layers in a given image. Our method is based on the ability to represent texture and cartoon layers as sparse combinations of atoms of predetermined Dictionaries. So if we want to fill simultaneously the image of texture which has unknown regions and image which consist of the cartoon image layer in that case we have to consider the MCA method to get the desired reconstructed image which providing the more promising result. The confidence pixels are going to help in both the cases to fill the incomplete image and reconstruct the both texture and cartoon image layer.

Advantage: This method is fusion of variation and regularization of the image that allows missing data and also automatically filling the missing holes in texture and also in the cartoon image layer.

Disadvantage: In this method there is necessity to consider the numerical solution to extend this approach. If the object has sparse representation then it creates the problem for this technique.

\section{Multiscale Image Modeling}

If we consider the nonseparable filter banks and direction based filter banks then we have to think differently because for this purpose it is not good idea to fully resemble on wavelet transform. To extend and to add this detail we have to consider the contourlet transform [9]. It can efficiently take control over the edges of image along with small number coefficient one dimensional contour because of its multiscale and directional properties. Contourlet transform region and its advantages helps author to inspect the image. The Detail study of contourlet coefficient makes clear idea about non-Gaussian trivial statics and strong dependencies. Contourlet coefficient is calculated about the Gaussian by considering the difficulty of neighboring coefficient magnitude. Technique is applied on the images which are affected by noise and the image where we have to retrieve the texture. While recovering the texture it shows too much improvement and additional things than wavelet transform and performance is also better.

Advantage: In denoising process the contourlet transform provides the better result than wavelet transform and in comparison to other technique it provides the good result in terms of human visual quality (HVS) and peal signal-to-noise ratio (PSNR).

Disadvantage: It is complex than the wavelet transforms and it also found difficulty to find the neighbouring coefficient.

\section{Image Completition with Patch Propagation}

This author described the necessities of semi-automatic image inpainting techniques. To complete this research the user plays the role of guide to help in the complete the structure and he found as favorer of the image [10]. This process works in two steps. In which first step user defined the region to be inpainted by drawing the object area border and physically specifies the missing information in the image. The border defined for that object move from the known region to unknown region. The patches are used to complete the texture in case of texture based synthesis. Author consider this problem as worldwide problem were he has to optimized the variety of structural and constancy constraints in that case the misplaced image patches produced all along the user defined curve. If we found the single curve in attendance then to get the optimum result we have to perform the dynamic programming. To produce the result with great accuracy dynamic programming importance is increased in this technique. The dealing with this propagation will decide the approximation of the result and how close is the result. In this way this technique are designed to complete the image which are damaged due to cracks, noise in the image, superimposed text, unknown object and each and every image inpainting techniques produces the good result or approximate result.

Advantage: Dynamic programming helps this algorithm to complete the image from single level to multilevel. If we able to found the single curve in this image then we can complete the image quickly and can also get the optimum result which is closely resemble with original image.

Disadvantage: For multiple objects the difficulty level is increased to optimize the result we have to propagate the technique to get approximate result.

\section{Discussion And Problems}

The above discussed techniques are providing the better result but they are also lacking in certain things. If we consider the size of object to recover, some of the techniques unable to produce the good result, because some of the techniques are designed for small image gaps only. If we complete the images with large gaps then it will give the result but the result quality will be poor and blurring effects also comes in act. Some of the techniques produce single resolution image result, to overcome that drawback multiresoultion approach is proposed. Wavelet transform is overcome by the contourlet transform for better result. PSNR values are used in some techniques which measured with the help of number of parts of images we created to complete the image.

The PSNR value is ratio of value decomposed image to value of the entire image which is calculated at the different level. This PSNR value satisfies the human visual system (HVS). After HVS is satisfy the image 
produce will be having maximum resemblance with original image. But this PSNR also creates problem when number of level increases.

\begin{tabular}{|c|l|l|c|}
\hline \multicolumn{5}{|c|}{ Table 1 Comparison of Techniques } \\
\hline Sr.No. & \multicolumn{1}{|c|}{ Author } & \multicolumn{1}{|c|}{ Proposed Method } & $\begin{array}{c}\text { Time } \\
\text { Efficiency }\end{array}$ \\
\hline 1 & M. Bertalmio & Image inpainting & $85 \%$ \\
\hline 2 & Timothy K. Shih & Multiresoultion Image Inpainting & $90 \%$ \\
\hline 3 & Iddo Drori & Fragment-Based Image Completion & $88.8 \%$ \\
\hline 4 & $\begin{array}{l}\text { Siddharth } \\
\text { Borikar }\end{array}$ & Completion of Images With Natural Scenes & $92.7 \%$ \\
\hline 5 & Zhang & Region Completion in single image & $87.9 \%$ \\
\hline 6 & Criminisis & Exemplar Based Inpainting & $96.2 \%$ \\
\hline 7 & Dongwook Cho & $\begin{array}{l}\text { Image Inpainting Based On Wavelet } \\
\text { Transform }\end{array}$ & $93.2 \%$ \\
\hline 8 & M. Elad & Image Inpainting Using MCA & $87.4 \%$ \\
\hline 9 & Duncan D.K. & $\begin{array}{l}\text { Image Inpainting Using Contourlet } \\
\text { Transform }\end{array}$ & $94.9 \%$ \\
\hline 10 & Jian et.al & Image Inpainting By Patch Propagation & $97 \%$ \\
\hline
\end{tabular}

\section{Conclusion}

In this paper a variety of image inpainting techniques are discussed which consist of PDE based Inpainting, Multiresoultion Image Inpainting, Fragment based Image completition, Completition of image with natural scenes, Exemplar based Inpainting etc. For each of the image detailed discussion consist of the working of the inpainting techniques which are used to fill out the missing regions also remove the unwanted object. From this detailed survey we discussed the advantages and shortcomings of all image inpainting techniques. Some of the techniques work only for small image gaps which overcome by the other inpainting technique. When we come across the removal of large region exemplar based inpainting provides the better result, this technique is design in such manner we can able to recover the object from small image gaps to large image gaps. This algorithm works for texture as well as structure synthesis. But this work with maximum accuracy when regions contains simple texture and structure. Contourlet transform technique design in such manner that it overcomes disadvantages of nearly all technique. Overall study tells that all technique trying to provide better result in terms of quality of the image and also trying to improve the efficiency in terms of time taken by image completition algorithm.

\section{References}

[1] M. Bertalmio, G. Sapiro, V. Caselles and C. Ballester, "image inpainting," In Proc. ACM Conf. Compo Graphics (SIGGRAPH), New Orleans, LU, 2000, pp. 417-424.

[2] Timothy K. Shih,Liang-Chen Lu, Ying-Hong Wang and Rong-Chi Chang, "Multi-resolution Image Inpainting" IEEE Transactions on Image Processing, 2003.

[3] D. Iddo, C. O. Daniel, and Y. Hezy, "Fragment Based Image Completion," ACM Transactions on Graphics, 2003, 22(3) 303-312.

[4] S, Borikar, K. K. Biswas, and S. Pattanaik, "Fast Algorithm for Completion of Images with Natural Scenes," graphics. Cs. ucf. eduiborikarlBorikarPaper. pdf.

[5] Y. Zhang, J Xiao, and M. Shah, "Region Completion in a Single Image,". EUROGRAPHICS, Grenoble, France, Short Presentations.

[6] A, Criminisi, P. Perez, and K. Toyama, "Region filling and object removal by exemplar-based image inpainting," IEEE Transactions on Image Processing, 2004, 13(9) 1200-1212.

[7] Dongwook cho and Tien D. Bui "Image Inpainting Using Wavelet-Based Inter-and Intra-Scale Depedency" IEEE Transactions on Image Processing, 2008.

[8] M. Elad, J.L. Starck, P. Querre, and D.L. Donoho, "Simultaneous Cartoon and texture image inpainting using morphological component analysis (MCA)," Journal on Applied and Computational Harmonic Analysis, 340-358, 2005.

[9] Po, Duncan D K, "Directional multiscale modeling of images using the contourlet transform", Statistical Signal Processing, 2003 IEEE Workshop on 28 Sept.-1 Oct. 2003.

[10] Z. Xu and S. Jian, "Image inpainting by patch propagation using patch sparsity," IEEE Transactions on Image Processing, Vol. 19, Pp. $1153-1165,2010$.

[11] A. A. Efros, and T. K Leung, 'Texture synthesis by non-parametric sampling," The Proceedings of the Seventh IEEE International Conference on Computer Vision, 1999, Vo1.2, pp. 1033-1038.

[12] L. W Wey, and W Levoy, "Fast texture synthesis using tree structured vector quantization," In Proc. ACM Conf. Computer Graphics (SIGGRAPH), 2000.

[13] M, Bertalmio, L. Vese, G. Sapiro, and S. Osher, "Simulaneous structure and texture image inpainting," IEEE Computer Society Conference on Computer Vision and Pattern Recognition, 2003,

Vol. 2, pp. 18-20.

[14] J Shen, X. Jin, C Zhou, and C C. L. Wang, "Gradient based image completion by solving the Poisson equation," Computer Graphics, 2007, 31(1) 119-126.

[15] S D. Rane, G. Spairo, and M. Bertalmio, "Structure and texture filling-in of missing image bloc.ks in wireless transmission and compression applications," IEEE Transactions on Image Processing, 2003, 12(3): 296-303. 